\title{
The Beat Goes On: Spontaneous Firing in Mammalian Neuronal Microcircuits
}

\author{
Michael Häusser, ${ }^{1}$ Indira M. Raman, ${ }^{2}$ Thomas Otis, ${ }^{3}$ Spencer L. Smith, ${ }^{3}$ Alexandra Nelson, ${ }^{4}$ Sascha du Lac, ${ }^{4}$ \\ Yonatan Loewenstein, ${ }^{5}$ Séverine Mahon, ${ }^{1}$ Cyriel Pennartz, ${ }^{6}$ Ivan Cohen, ${ }^{7,8}$ and Yosef Yarom ${ }^{9}$ \\ ${ }^{1}$ Wolfson Institute for Biomedical Research and Department of Physiology, University College London, London WC1E 6BT, United Kingdom, ${ }^{2}$ Department \\ of Neurobiology and Physiology, Northwestern University, Evanston, Illinois 60208, ${ }^{3}$ Department of Neurobiology, University of California Los Angeles \\ Medical Center, Los Angeles, California 90095-1763, ${ }^{4}$ Systems Neurobiology Laboratory, The Salk Institute, La Jolla, California 92037, ${ }^{5}$ Department of Brain \\ and Cognitive Sciences, Massachusetts Institute of Technology, Cambridge, Massachusetts 01239, ${ }^{\circ}$ Department of Animal Physiology and Cognitive \\ Neuroscience, University of Amsterdam, Amsterdam 1090 GB, The Netherlands, 'Institut National de la Santé et de la Recherche Médicale (INSERM), \\ Equipe Mixte de l'INSERM 224, University of Paris, Centre Hospitalier Universitairé Pitié Salpêtrière, Paris 75013, France, ${ }^{8}$ Department of Neuroscience, \\ Baylor College of Medicine, Houston, Texas 77030, and ${ }^{9}$ Department of Neurobiology, Hebrew University, Edmond Safra Campus, Jerusalem 91904, Israel
}

Key words: action potential; channel; cerebellum; pacemaker; hippocampus; neuromodulation

Many neurons in the brain remain active even when an animal is at rest. Over the past few decades, it has become clear that, in some neurons, this activity can persist even when synaptic transmission is blocked and is thus endogenously generated. This "spontaneous" firing, originally described in invertebrate preparations (Alving, 1968; Getting, 1989), arises from specific combinations of intrinsic membrane currents expressed by spontaneously active neurons (Llinas, 1988). Recent work has confirmed that, far from being a biophysical curiosity, spontaneous firing plays a central role in transforming synaptic input into spike output and encoding plasticity in a wide variety of neural circuits. This mini-symposium highlights several key recent advances in our understanding of the origin and significance of spontaneous firing in the mammalian brain.

Biophysical mechanisms underlying spontaneous activity To maintain spontaneous activity, intrinsic currents must interact to depolarize the cell membrane to threshold, elicit an action potential, and repolarize the membrane to negative potentials from which the next spike can be initiated. Several studies have identified currents responsible for the subthreshold depolarizations leading to each spike. In some neurons, this depolarization results from dynamic recruitment and activation of hyperpolarizationactivated channels and T-type Ca channels (McCormick and Huguenard, 1992). In other neurons, voltage-gated Na currents and/or nonselective cation currents bring the membrane potential to threshold (Raman et al., 2000; Taddese and Bean, 2002; Do and Bean, 2003; Jackson et al., 2004).

In spontaneously active neurons exhibiting high firing rates, an additional issue arises regarding the availability of $\mathrm{Na}$ channels, which usually inactivate substantially during each action

Received Aug. 17, 2004; accepted Aug. 24, 2004.

We thank Richard Miles for comments on this manuscript.

Correspondence should be addressed to Michael Häusser, Wolfson Institute for Biomedical Research, University

College London, Gower Street, London WC1E 6BT, UK. E-mail: m.hausser@ucl.ac.uk.

DOI:10.1523/JNEUROSCI.3375-04.2004

Copyright $\odot 2004$ Society for Neuroscience $\quad$ 0270-6474/04/249215-05\$15.00/0 potential. In many neurons, recovery from inactivation is incomplete during brief interspike intervals at moderately hyperpolarized potentials. After a burst of high-frequency action potentials, such cells therefore become refractory and stop firing until $\mathrm{Na}$ channels are allowed to recover. In contrast, in neurons that maintain regular spontaneous firing for hundreds of consecutive action potentials, recovery between spikes must be fast enough to compensate for inactivation during spikes. In cerebellar Purkinje neurons, whose spontaneous firing rates are unusually high ( $\sim 40-50 \mathrm{~Hz}$ ) (Häusser and Clark, 1997; Womack and Khodakhah, 2002; Smith and Otis, 2003), this issue is partly resolved by Na channels with "resurgent" kinetics (Raman and Bean, 1997) (Fig. 1). With depolarization, these voltage-gated, tetrodotoxin (TTX)-sensitive Na channels open briefly and then become blocked by a voltage-dependent, open-channel blocker. This endogenous blocking protein not only limits inactivation by competing with the fast inactivation gate but also unbinds rapidly at negative potentials. Consequently, a resurgent $\mathrm{Na}$ current flows briefly as channels unblock (open) with repolarization. Significantly, the channels then deactivate into closed states that are readily available for reactivation, thereby shortening refractory periods and facilitating high-frequency spontaneous firing ( $\mathrm{Ra}$ man and Bean, 2001; Grieco et al., 2002; Khaliq et al., 2003). Which $\mathrm{Na}$ channel $\alpha$ subunits are targeted by the blocker? In Purkinje neurons, fast inactivation proceeds somewhat more slowly in $\mathrm{Na}_{\mathrm{V}} 1.6$ channels than in other $\alpha$ subunits, such that $\mathrm{Na}_{\mathrm{V}} 1.6$ channels have a much higher chance of binding the blocker than the fast inactivation gate. As a consequence, $\mathrm{Na}_{\mathrm{V}} 1.6$ channels are more susceptible to block and unblock than other $\alpha$ subunits, thereby carrying most of the resurgent $\mathrm{Na}$ current of Purkinje cells (Raman et al., 1997). In other neurons, however, substantial resurgent $\mathrm{Na}$ currents can flow through non- $\mathrm{Na}_{\mathrm{V}} 1.6$ $\alpha$ subunits, indicating that, under the right conditions, the blocker (or blockers) can interact with a variety of $\mathrm{Na}$ channels (Do and Bean, 2004; Grieco and Raman, 2004). Thus, the expression of a blocking protein, whose molecular identity is under active investigation, endows otherwise classical $\mathrm{Na}$ channel $\alpha$ 

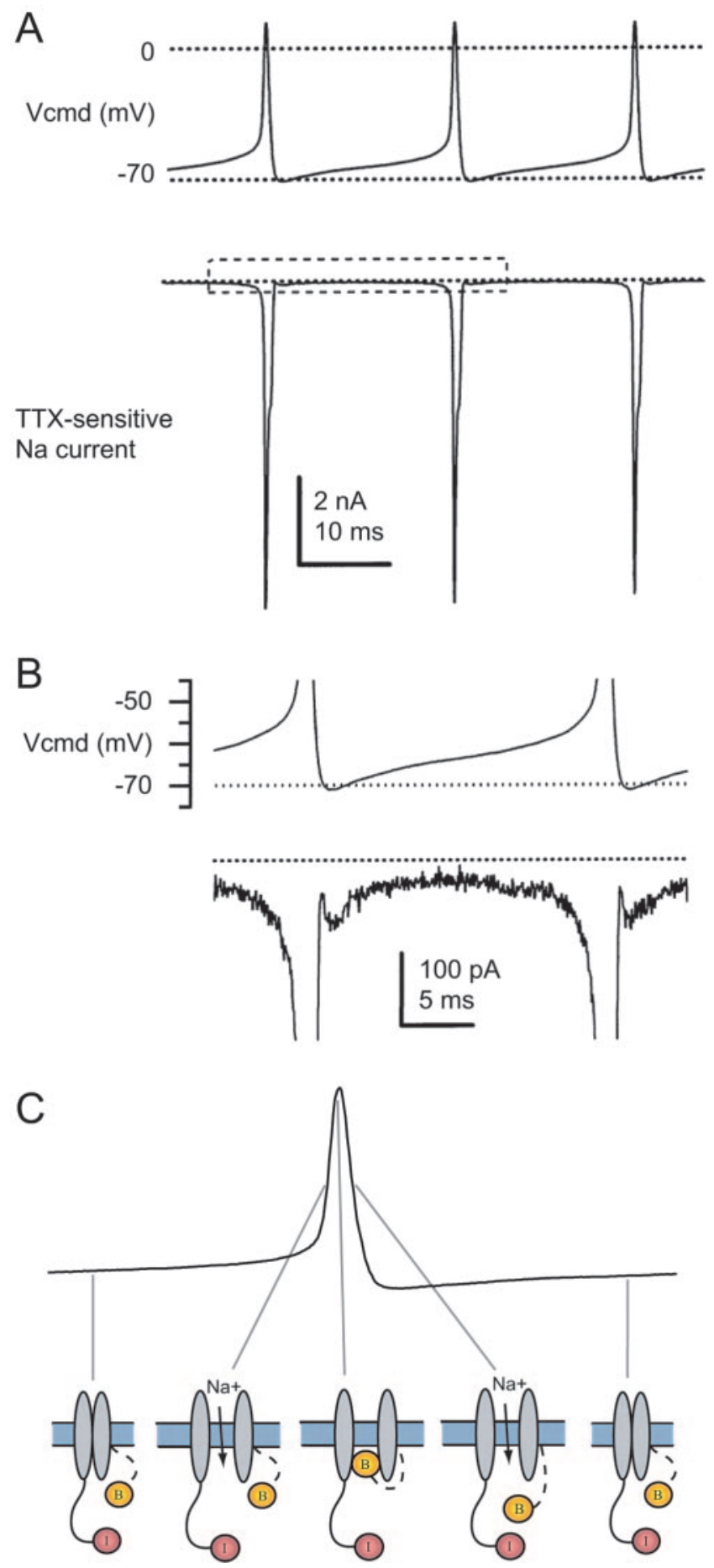

Figure 1. Ionic currents underlying spontaneous firing. A, Spontaneous firing in an isolated Purkinje cell (top trace). Bottom traces show underlying TTX-sensitive voltage-gated Na current activated during spiking by applying the top trace as a voltage-clamp command ( $\mathrm{Vcmd}$ ). Modified from Raman and Bean (1999). B, Close-up of Na current activated at subthreshold potentials, with resurgent $\mathrm{Na}$ current apparent on the falling phase of the action potential (dashed box in A). Modified from Raman and Bean (1999). C, Schematic illustration of Na channel gating during a single action potential. Gray lines indicate the approximate times and voltages during the spike that favor each conformation. Channels begin in the closed state, with the inactivation gate $(I)$ and the blocking particle $(B)$ unbound. As the spike proceeds, channels open, block, unblock (producing resurgent current), and close. Note that the binding of the inactivation gate can be prevented by binding of the blocking particle. Modified from Grieco et al. (2002). subunits with distinctive, apparently physiologically relevant, properties.

\section{Plasticity of spontaneous firing in cerebellar Purkinje neurons}

The rapid regular firing of Purkinje neurons can thus be primarily accounted for by the macroscopic kinetics of their intrinsic voltage-gated channels, operating on a timescale of milliseconds. On a longer timescale, however, this spontaneous firing can be accelerated and slowed, or even turned on and off. Recent work by Smith and Otis (2003) has shown that long-term changes in intrinsic Purkinje neuron firing rates can occur in an activitydependent manner. This mechanism is notable in that it constitutes a form of circuit plasticity that does not rely on changing synaptic strength. The changes in Purkinje neuron firing have been traced to modulation by the nitric oxide-cGMP signaling cascade (Fig. 2A). Although the role of this pathway in the vascular system is well established, its importance for signaling in the brain has been the subject of much debate. Components of the cascade are expressed throughout the brain, but they seem to be particularly important in the cerebellar cortex, in which they are especially abundant. Nitric oxide synthase is present in the granule neurons and in their axon terminals, the parallel fibers as well as in basket cells, a class of inhibitory interneurons (Bredt et al., 1990). Soluble guanylate cyclase, the canonical target for nitric oxide, is highly expressed in Purkinje neurons (Ariano et al., 1982). In addition, several potential targets of cGMP are present in Purkinje neurons, including cGMP-dependent protein kinase (PKG), cGMP-gated ion channels, and cGMP phosphodiesterase (Lohmann et al., 1981; el-Husseini et al., 1995; Bellamy and Garthwaite, 2001). Not surprisingly, cerebellar cortex has proven to be a model preparation for examining nitric oxide-cGMP physiology in the brain. Depolarizations cause biochemically detectable elevations of nitric oxide-cGMP, and stimulation of parallel fibers causes production of nitric oxide (Shibuki and Kimura, 1997). Despite this wealth of knowledge about the expression and biochemistry of this signaling cascade, there is controversy regarding its physiological roles in the cerebellum. Recent results (Smith and Otis, 2003) indicate that nitric oxide released by stimulation of parallel fibers in cerebellar slices diffuses to Purkinje neurons, in which it activates soluble guanylate cyclase. The resulting rise in cGMP activates the cGMPdependent protein kinase (PKG) and increases firing rates in the Purkinje neurons, presumably by phosphorylation of target proteins such as ion channels. Current efforts are being focused on identifying the contribution of several candidate conductances to the modulation of firing rate, particularly $\mathrm{Ca}$-activated $\mathrm{K}$ currents (Womack and Khodakhah, 2002) and resurgent Na currents (Raman and Bean, 2001; Grieco et al., 2002; Khaliq et al., 2003).

\section{Bistability of Purkinje cell output}

In addition to being finely modulated over long timescales, can spontaneous spiking in Purkinje cells also be turned on and off? In vitro studies have revealed that the membrane potential of Purkinje cells can display intrinsic bistability, in which the hyperpolarized state is quiescent and the depolarized state is characterized by persistent high-frequency spiking (Llinas and Sugimori, 1980; Williams et al., 2002). How does this relate to the firing pattern of Purkinje cells in vivo? Recent work has demonstrated that the membrane potential and spike output of Purkinje cells can also exhibit bistability in vivo. This bistability of the membrane potential implies that the instantaneous firing rate is not 
A

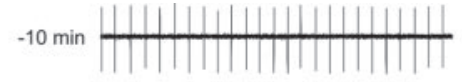

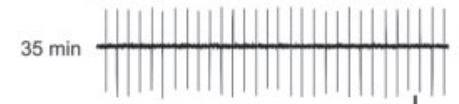

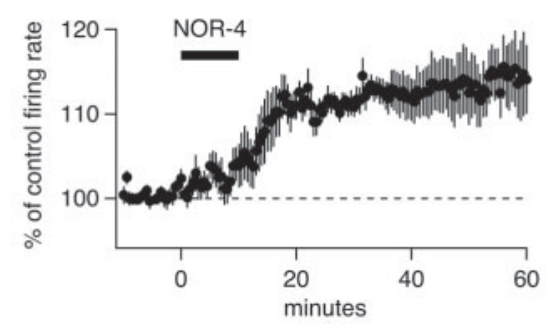

B

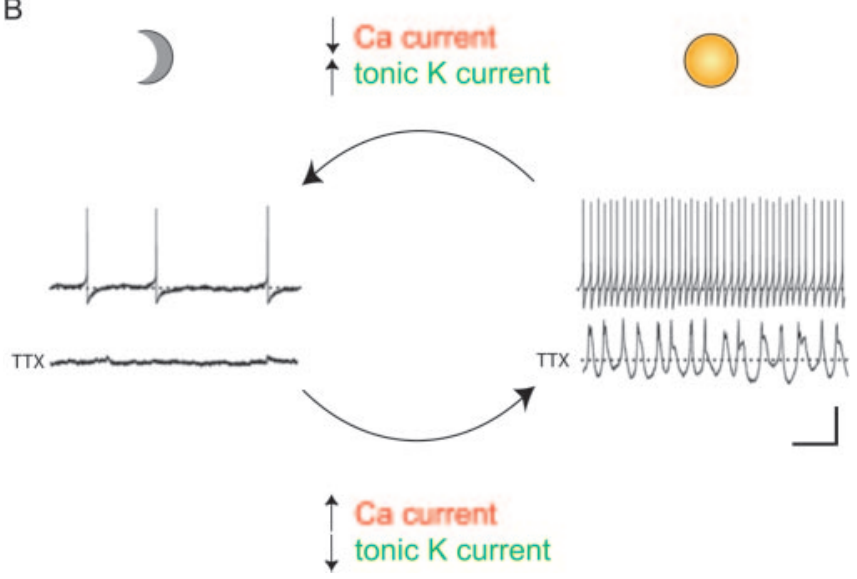

Figure 2. Plasticity of spontaneous firing. A, Persistent increase in spontaneous firing rate in cerebellar Purkinje cells triggered by application of the nitric oxide donor NOR-4. Top shows traces of spontaneous firing in a cell-attached recording from a Purkinje cell in a cerebellar slice $10 \mathrm{~min}$ before and $35 \mathrm{~min}$ after NOR-4 application. Calibration: 75 pA, $100 \mathrm{msec}$. Bottom shows time course of changes in average firing rate caused by NOR-4 for six Purkinje neurons. Modified from Smith and Otis (2003). B, Schematic illustration showing circadian regulation of spontaneous firing in $\mathrm{SCN}$ neurons. Left, At night, spontaneous firing rate is low (dashed line, -59 $\mathrm{mV}$ ), and TTX fails to uncover membrane potential oscillations ( $-59 \mathrm{mV}$ membrane potential). Right, During the day, spontaneous firing rate is high (dashed line, $-54 \mathrm{mV}$ ), and TTX reveals a depolarized membrane potential (dashed line, $-41 \mathrm{mV}$ ), exhibiting oscillations. These changes are attributable to modulation of L-type $\mathrm{Ca}$ current and tonic $\mathrm{K}$ current. Calibration: spiking traces, $500 \mathrm{msec}, 40 \mathrm{mV}$; TTX traces, $500 \mathrm{msec}, 20 \mathrm{mV}$. For details, see Pennartz et al. (2002).

only a function of the instantaneous synaptic inputs but also of the past inputs that control the state of the cell. Surprisingly, the state of Purkinje cells is often determined by input from the climbing fiber pathway, which generates a stereotypic discharge pattern known as a complex spike at a mean rate of $\sim 1 \mathrm{~Hz}$ (Ito, 1984). Activation of climbing fibers by sensory input while the cell is in a hyperpolarized quiescent state often generates a sustained depolarization accompanied by high-frequency simple spike activity. Conversely, the same climbing fiber input delivered while the cell is in the active depolarized state can induce a transition to the hyperpolarized state (Y. Loewenstein and S. Mahon, unpublished data). This behavior can be captured by a simple dynamical model and is similar to a toggle switch in electronics. The emerging picture is far from complete, but clearly indicates that the common assumption that simple spike activity reflects the instantaneous activation of the parallel fibers is an oversimplification (Ghez and Thach, 2000) and that climbing fiber input, in addition to parallel fiber input, plays an important role in determining the firing pattern of simple spikes in cerebellar Purkinje cells.

\section{Long-term modulation of spiking by inhibition in the vestibular nuclei}

Like Purkinje neurons, their target neurons in the vestibular nucleus are also spontaneously active. Vestibular nucleus neurons, which participate in the circuit regulating the vestibulo-ocular reflex (VOR), change their spontaneous and stimulus-evoked firing rates in conjunction with behavioral plasticity in the VOR ( du Lac, 1995; Darlington et al., 2002). Although these changes in vestibular neuron firing are commonly assumed to reflect synaptic plasticity, recent evidence suggests a role for use-dependent changes in intrinsic excitability. Persistent increases in both spontaneous and evoked firing in vestibular nucleus neurons can be induced by brief periods of synaptic inhibition or direct membrane hyperpolarization (Nelson et al., 2003). This new form of cellular plasticity, termed firing rate potentiation (FRP), provides a candidate mechanism for transforming synaptic inhibition from Purkinje neurons into behavioral changes in the VOR.

How does synaptic inhibition induce persistent increases in intrinsic excitability? Although elevations in intracellular calcium are associated with most other forms of cellular plasticity, imaging experiments indicate that intracellular $\mathrm{Ca}$ levels are reduced in vestibular nucleus neurons during membrane hyperpolarization. Correspondingly, firing rate potentiation can be induced by blocking voltage-gated $\mathrm{Ca}$ channels or by transiently replacing extracellular Ca with $\mathrm{Mg}$, consistent with the idea that synaptic inhibition triggers FRP by reducing intracellular Ca levels. The signaling pathways that couple reductions in Ca to increases in excitability are under active investigation and are likely to involve calcium/calmodulin-dependent protein kinase II (CaMKII) and BK-type Ca-activated K currents. Blockade of CaMKII, which is constitutively active in vestibular nucleus neurons, produces rapid increases in intrinsic excitability via reductions in BK-type Ca-activated K currents (Smith et al., 2002). After the induction of firing rate potentiation, BK currents are reduced in vestibular nucleus neurons, and previous blockade of BK currents occludes firing rate potentiation (Nelson et al., 2003). Thus, in contrast with quiescent CNS neurons, which change their properties in response to excitatory drive and concomitant increases in calcium influx, the excitability of spontaneously firing vestibular nucleus neurons can be regulated by synaptic inhibition and decreases in calcium influx. Identification of the underlying mechanisms will enable investigations into the role of firing rate potentiation in behavioral plasticity of the VOR in genetically modified mice.

\section{The rise and fall of spontaneous firing in the mammalian biological clock}

High-frequency spontaneous firing is thus typical of many cerebellar neurons and their targets, as well as neurons in other motor centers, and may well play an important role in regulating motor control. Low-frequency spontaneous firing (at rates of $<10 \mathrm{~Hz}$ ) has been identified in neurons in brain regions that participate in homeostatic control. This is exemplified by the suprachiasmatic nucleus (SCN), because the firing rate of SCN neurons is clearly linked to behavioral and physiological output. The SCN has been recognized as the central circadian pacemaker of the mammalian brain and imposes its output onto target structures in the hypothalamus, thalamus, and other areas by way of a diurnal modulation of spontaneous discharge frequency (Schwartz et al., 1987). This diurnal rhythm originates from a cell-autonomous 
process, being driven by molecular loops within clock cells that involve transcription, translation, and protein-mediated negative feedback acting on gene expression (Reppert and Weaver, 2001). One of the key issues in this field is to elucidate how membrane mechanisms transduce intracellular clock signals into a synchronized firing rate output that is broadcast to the rest of the brain and body.

To achieve progress in this area, two questions have to be addressed. First, which ionic channels regulate spontaneous firing rate of SCN neurons per se, regardless of circadian aspects? Second, which of these ionic mechanisms is subject to diurnal modulation, and how does this modulation contribute to an enhanced firing rate during the day and a decline in spontaneous firing during the night? Knowledge about spontaneous firing mechanisms has steadily accumulated over the past two decades and has highlighted the role of slowly inactivating $\mathrm{Na}$ currents and fast-activating K currents in shaping the membrane potential trajectories preceding and succeeding action potentials on a fast timescale (Bouskila and Dudek, 1995; Pennartz et al., 1997). However, with respect to the second question, it has proved to be a challenge to identify the ionic mechanisms responsible for regulating action potential generation on a slow timescale, stretching an average spike interval of $\sim 120 \mathrm{msec}$ during the peak of daytime firing to $\sim 400-1000 \mathrm{msec}$ during the night (Fig. $2 B$ ). The importance of slow firing rate regulation in the SCN prompted us to investigate the tonic spike-independent electrophysiological behavior of SCN neurons, using perforated-patch recordings in SCN slices in the presence of TTX. These experiments revealed two remarkable day-night differences: day cells were tonically depolarized with respect to night cells and exhibited spontaneous oscillations in membrane potential at a frequency of 2-7 Hz. These oscillations appeared to phase-lock to action potentials and were mediated by L-type Ca channels (Pennartz et al., 2002). The tonic membrane depolarization in day cells persisted when L-type Ca channels were blocked, despite the disappearance of oscillations, indicating the presence of at least two ionic mechanisms regulating diurnal rhythmicity. In accordance with the strong day-night difference in membrane potential oscillations, the amplitude of L-type Ca currents differed between the day and night phase. As predicted, blockade of L-type current exerted a differential day-night effect on spontaneous firing patterns (Pennartz et al., 2002; Ikeda et al., 2003). The molecular identity of the ionic current mediating the functionally dissociable tonic membrane depolarization during the daytime remains unknown. The Ca currents involved in expressing rhythmicity may also in turn regulate intracellular signaling, because day-night differences in intracellular calcium levels have been observed (Colwell, 2000; Ikeda et al., 2003). However, this issue requires additional investigation because at least one component of cytosolic Ca rhythmicity is not blocked by an L-type Ca channel antagonist (Ikeda et al., 2003).

\section{Linking single-cell spontaneous firing to population activity}

What is the relationship between the activity of single spontaneously active neurons and their neighbors in a network with recurrent excitation and inhibition? The hippocampal CA3 pyramidal cell network is a good model system to study this question and is particularly relevant because this region can initiate seizure activity. In a recent study (Cohen and Miles, 2000), the spontaneous activity of a population of several hundred CA3 neurons was studied non-invasively using extracellular multiunit recordings. This technique showed that the CA3 region of the hippocampus is spontaneously active, at a mean frequency for each cell of $\sim 0.1-0.2 \mathrm{~Hz}$ (Cohen and Miles, 2000). What is the relative contribution of intrinsic cellular mechanisms and excitatory and inhibitory synapses to this activity? When both inhibitory and excitatory synaptic transmission are blocked, spontaneous firing almost doubles, indicating that single CA3 neurons can generate action potentials endogenously at a low rate of $\sim 0.2-0.4 \mathrm{~Hz}$. The activity of inhibitory neurons, either intrinsic or driven by recurrent collaterals of the pyramidal cells, thus strongly brakes CA3 population activity and prevents synchronized firing. Surprisingly, excitatory transmission only accounts for a moderate increase in population firing. This small effect seems paradoxical but results from a functional antagonism between excitatory transmission at synapses between pyramidal cells and onto inhibitory interneurons.

Although this spontaneous CA3 population activity often seems relatively unpatterned, blocking inhibition in slices from healthy animals increases activity levels and leads to bursts of activity separated by long silences, which has been compared with interictal activity (Hill et al., 1973). A similar pattern of spontaneous interictal bursting is generated by the subiculum of slices obtained after therapeutic hippocampal resection from human patients with mesial temporal lobe epilepsy (Kohling et al., 1998). However, in this tissue, GABAergic transmission enhances rather than suppresses spontaneous activity. Comparison of single-cell and population activities shows that three populations of cells exist in the epileptic focus (Cohen et al., 2002). Interneurons and a minority of pyramidal cells fire during interictal population bursts, although most pyramidal cells are strongly inhibited. GABA has depolarizing actions in a minority of pyramidal cells attributable to a pathologically elevated intracellular $\mathrm{Cl}$ concentration. This seemingly paradoxical role of inhibitory transmission in epileptic tissue is reminiscent of its action in the developing brain, in which spontaneous patterned population activity is ubiquitous and contributes critically to circuit formation (Penn and Shatz, 1999). Understanding the genesis and regulation of spontaneous firing in hippocampal pyramidal cells may provide important clues to understanding the transition between normal patterns of activity and pathophysiological synchronization in epilepsy.

\section{Summary}

Although spontaneous firing was originally described decades ago, investigation of its underlying molecular and biophysical mechanisms and awareness of its potential functional consequences has seen a dramatic resurgence in the past few years. This is attributable to development of new techniques for investigating channel function in isolated neurons (cloning, identification, and localization of ion channels that underlie spontaneous activity) and systems approaches for understanding the contribution of single neurons to network function. Results emerging from these techniques have led to a growing consensus that spontaneous firing is not only a prominent feature of many neuronal networks but may also serve useful functional roles, contributing to regulating information flow in different microcircuits in the brain.

\section{References}

Alving BO (1968) Spontaneous activity in isolated somata of Aplysia pacemaker neurons. J Gen Physiol 51:29-45.

Ariano MA, Lewicki JA, Brandwein HJ, Murad F (1982) Immunohistochemical localization of guanylate cyclase within neurons of rat brain. Proc Natl Acad Sci USA 79:1316-1320.

Bellamy TC, Garthwaite J (2001) “cAMP-specific" phosphodiesterase con- 
tributes to cGMP degradation in cerebellar cells exposed to nitric oxide. Mol Pharmacol 59:54-61.

Bouskila Y, Dudek FE (1995) A rapidly activating type of outward rectifier $\mathrm{K}^{+}$current and A-current in rat suprachiasmatic nucleus neurones. J Physiol (Lond) 488:339-350.

Bredt DS, Hwang PM, Snyder SH (1990) Localization of nitric oxide synthase indicating a neural role for nitric oxide. Nature 347:768-770.

Cohen I, Miles R (2000) Contributions of intrinsic and synaptic activities to the generation of neuronal discharges in in vitro hippocampus. J Physiol (Lond) 524:485-502.

Cohen I, Navarro V, Clemenceau S, Baulac M, Miles R (2002) On the origin of interictal activity in human temporal lobe epilepsy in vitro. Science 298:1418-1421.

Colwell CS (2000) Circadian modulation of calcium levels in cells in the suprachiasmatic nucleus. Eur J Neurosci 12:571-576.

Darlington CL, Dutia MB, Smith PF (2002) The contribution of the intrinsic excitability of vestibular nucleus neurons to recovery from vestibular damage. Eur J Neurosci 15:1719-1727.

Do MT, Bean BP (2003) Subthreshold sodium currents and pacemaking of subthalamic neurons: modulation by slow inactivation. Neuron 39:109-120.

Do MT, Bean BP (2004) Sodium currents in subthalamic nucleus neurons from Nav1.6-null mice. J Neurophysiol 92:726-733.

du Lac S (1995) Learning and memory in the vestibulo-ocular reflex. Annu Rev Neurosci 18:409-441.

el-Husseini AE, Bladen C, Vincent SR (1995) Expression of the olfactory cyclic nucleotide gated channel (CNG1) in the rat brain. NeuroReport 6:1459-1463.

Getting PA (1989) Emerging principles governing the operation of neural networks. Annu Rev Neurosci 12:185-204.

Ghez C, Thach WT (2000) The cerebellum. In: Principles of neural science, Ed 4 (Kandel ER, Schwartz JH, Jessell TM, eds), pp 832-852. New York: McGraw-Hill.

Grieco TM, Raman IM (2004) Production of resurgent current in NaV1.6null Purkinje neurons by slowing sodium channel inactivation with betapompilidotoxin. J Neurosci 24:35-42.

Grieco TM, Afshari FS, Raman IM (2002) A role for phosphorylation in the maintenance of resurgent sodium current in cerebellar Purkinje neurons. J Neurosci 22:3100-3107.

Häusser M, Clark BA（1997) Tonic synaptic inhibition modulates neuronal output pattern and spatiotemporal synaptic integration. Neuron 19:665-678.

Hill RG, Simmonds MA, Straugn DW (1973) A comparative study of some convulsant substances as gamma-aminobutyiric acid antagonists in the feline cerebral cortex. Br J Pharmacol 49:37-51.

Ikeda M, Sugiyama T, Wallace CS, Gompf HS, Yoshioka T, Miyawaki A, Allen CN (2003) Circadian dynamics of cytosolic and nuclear $\mathrm{Ca}^{2+}$ in single suprachiasmatic nucleus neurons. Neuron 38:253-263.

Ito M (1984) The cerebellum and neural control. New York: Raven.

Jackson AC, Yao GL, Bean BP (2004) Mechanism of spontaneous firing in dorsomedial suprachiasmatic nucleus neurons. J Neurosci 24:7985-7998.

Khaliq ZM, Gouwens NW, Raman IM (2003) The contribution of resurgent sodium current to high-frequency firing in Purkinje neurons: an experimental and modeling study. J Neurosci 23:4899-4912.

Kohling R, Lucke A, Straub H, Speckmann EJ, Tuxhorn I, Wolf P, Pannek H, Oppel F (1998) Spontaneous sharp waves in human neocortical slices excised from epileptic patients. Brain 1998 121:1073-1087.

Llinas RR (1988) The intrinsic electrophysiological properties of mammalian neurons: insights into central nervous system function. Science 242:1654-1664.
Llinas R, Sugimori M (1980) Electrophysiological properties of in vitro Purkinje cell somata in mammalian cerebellar slices. J Physiol (Lond) 305:171-195.

Lohmann SM, Walter U, Miller PE, Greengard P, De Camilli P (1981) Immunohistochemical localization of cyclic GMP-dependent protein kinase in mammalian brain. Proc Natl Acad Sci USA 78:653-657.

McCormick DA, Huguenard JR (1992) A model of the electrophysiological properties of thalamocortical relay neurons. J Neurophysiol 68:1384-1400.

Nelson AB, Krispel CM, Sekirnjak C, du Lac S (2003) Long-lasting increases in intrinsic excitability triggered by inhibition. Neuron 40:609-620.

Penn AA, Shatz CJ (1999) Brain waves and brain wiring: the role of endogenous and sensory-driven neural activity in development. Pediatr Res 45:447-458.

Pennartz CMA, Bierlaagh MA, Geurtsen AMS (1997) Cellular mechanisms underlying spontaneous firing in rat suprachiasmatic nucleus: involvement of a slowly inactivating component of sodium current. J Neurophysiol 78:1811-1825.

Pennartz CMA, De Jeu MTG, Bos NPA, Schaap J, Geurtsen AMS (2002) Diurnal modulation of pacemaker potentials and calcium current in the mammalian circadian clock. Nature 416:286-290.

Raman IM, Bean BP (1997) Resurgent sodium current and action potential formation in dissociated cerebellar Purkinje neurons. J Neurosci 17:4517-4526.

Raman IM, Bean BP (1999) Ionic currents underlying spontaneous action potentials in isolated cerebellar Purkinje neurons. J Neurosci 19:1663-1674.

Raman IM, Bean BP (2001) Inactivation and recovery of sodium currents in cerebellar Purkinje neurons: evidence for two mechanisms. Biophys 80:729-737.

Raman IM, Sprunger LK, Meisler MH, Bean BP (1997) Altered subthreshold sodium currents and disrupted firing patterns in Purkinje neurons of Scn8a mutant mice. Neuron 19:881-891.

Raman IM, Gustafson AE, Padgett D (2000) Ionic currents and spontaneous firing in neurons isolated from the cerebellar nuclei. J Neurosci 20:9004-9016.

Reppert SM, Weaver DR (2001) Molecular analysis of mammalian circadian rhythms. Annu Rev Physiol 63:647-676.

Schwartz WJ, Gross RA, Morton MT (1987) The suprachiasmatic nucle contain a tetrodotoxin-resistant circadian pacemaker. Proc Natl Acad Sci USA 84:1694-1698.

Shibuki K, Kimura S (1997) Dynamic properties of nitric oxide release from parallel fibers in rat cerebellar slices. J Physiol (Lond) 498:443-452.

Smith MR, Nelson AB, Du Lac S (2002) Regulation of firing response gain by calcium-dependent mechanisms in vestibular nucleus neurons. J Neurophysiol 87:2031-2042.

Smith SL, Otis TS (2003) Persistent changes in spontaneous firing of Purkinje neurons triggered by the nitric oxide signaling cascade. J Neurosci 23:367-372.

Taddese A, Bean BP (2002) Subthreshold sodium current from rapidly inactivating sodium channels drives spontaneous firing of tuberomammillary neurons. Neuron 33:587-600.

Williams SR, Christensen SR, Stuart GJ, Häusser M (2002) Membrane potential bistability is controlled by the hyperpolarization- activated current $\mathrm{I}_{\mathrm{h}}$ in rat cerebellar Purkinje neurons in vitro. J Physiol (Lond) 539:469-483.

Womack M, Khodakhah K (2002) Active contribution of dendrites to the tonic and trimodal patterns of activity in cerebellar Purkinje neurons. J Neurosci 22:10603-10612. 